\title{
Towards explanation of pulsar radio spectra
}

\author{
S. A. Petrova ${ }^{\star}$ \\ Institute of Radio Astronomy, Chervonopraporna St. 4, 61002 Kharkov, Ukraine
}

Received 15 June 2001 / Accepted 11 December 2001

\begin{abstract}
Refraction of radio waves in the ultrarelativistic highly magnetized pulsar plasma is considered. The influence of the effect on the observed pulsar spectra is studied. It is shown that refraction can cause a marked redistribution of the intensity in the emission beam. For a given sight-line trajectory across the beam this affects not only the apparent profile morphology but also the total intensity detected. As refraction is frequency-dependent, it can modify the pulsar spectrum. The spectral changes are peculiar to both the core and conal components. For the typical parameters of the magnetospheric plasma the effect is found to be efficient at the frequencies above $1 \mathrm{GHz}$. It is demonstrated that refraction can lead to the high-frequency steepening of the core-component spectrum with the subsequent flattening at still higher frequencies. In addition, the effect can account for the inversion of the intensity ratio of the conal components and the consequent break in the spectra of the conal-double pulsars. The high-frequency features of the pulsar spectra are compared with the other observational consequences of refraction. The conclusion is made that at the frequencies above $1 \mathrm{GHz}$ the properties of pulsar radiation are mainly determined by the propagation effects in the magnetospheric plasma.
\end{abstract}

Key words. plasmas - waves - pulsars: general

\section{Introduction}

Nowadays pulsars are observed over three decades in radio frequency, from a few times $10 \mathrm{MHz}$ to a few times $10 \mathrm{GHz}$, and their radio spectra show the following features (e.g. Malofeev 1999). At the intermediate frequencies, from a few hundred $\mathrm{MHz}$ to a few $\mathrm{GHz}$, the spectra are typically represented by a power law with the spectral index $\sim 1-2$. At the lower frequencies one observes a turn-over in the spectrum of some pulsars. At the higher frequencies the power law often steepens, with the spectral index being $\sim 2-3$. At extremely high frequencies $(\sim 30 \mathrm{GHz})$ in some pulsars that are still observable, the spectrum tends to flatten or even shows a turn-up (Wielebinski et al. 1993; Kramer et al. 1996).

Of course, pulsar spectra are primarily determined by the emission mechanism, but they can also be affected by the propagation effects in the magnetospheric plasma and the effects of observational geometry. In the absence of a commonly accepted concept of the radio emission mechanism, it is reasonable to examine the role of these effects in the formation of the observed spectra in order to ascertain what features are actually intrinsic to the emission mechanism.

\footnotetext{
* e-mail: rai@ira.kharkov.ua
}

The low-frequency turn-over in the pulsar spectrum is often attributed to the propagation effects, e.g. to free-free absorption (Malov 1979) or induced scattering (Lyubarskii \& Petrova 1996) of the radio waves by the particles of the magnetospheric plasma. As is shown by Sieber (1997), the high-frequency break in the spectrum can be caused by geometrical effects. In that paper an artificial emission beam composed of three Gaussian components was considered. Given the non-central cut of the emission cone by the sight line, the high-frequency shrinkage of the cone implies that increasingly greater part of the emission beam becomes invisible, so that the core component weakens as compared to the conal outriders and the total spectrum can steepen. It is essential that the difference in the spectral behaviour of the profile components can be explained regardless of the properties of the emission mechanism. Note that an idea of a single emission mechanism for all the profile components indeed seems to be preferable (e.g. Lyne \& Manchester 1988).

Furthermore, the component structure of complex profiles is not necessarily the attribute of the emission mechanism itself: The rays can group into separate components owing to refraction in pulsar plasma. It has been already demonstrated that taking into account refraction allows to explain the diversiform morphology of the observed pulse profiles within the frame of a primordial 
hollow-cone emission model (Petrova \& Lyubarskii 2000; Petrova 2000). Note that the hollow-cone model is believed to be physically grounded, if the emission process is associated with pulsar secondary plasma. Indeed, close to the magnetic axis the field lines of a dipolar field are nearly straight and the electron-positron cascade does not develop, so that there is the lack of plasma in the central part of the open field line tube (Arons \& Scharlemann 1979). Smooth variation of the plasma number density across the tube implies that the density should decrease towards both the magnetic axis and the tube edges.

Refraction acts to decline the rays in the direction of the plasma density decrease. Therefore the rays emitted in the inner part of the tube deviate towards the magnetic axis and form the core component, whereas those originating in the outer tube part deviate outwards and form a pair of conal outriders. Thus, it is refraction that hides the rays in the central part of the emission beam, which is invisible given the non-central sight-line trajectory. Provided that the radius-to-frequency mapping is the case, at higher frequencies refraction becomes stronger (e.g. Lyubarskii \& Petrova 1998). Then the grouping of the core rays is reinforced and the resultant spectrum is even steeper than the spectrum, which is determined purely by the geometrical effects.

At still higher frequencies the inner rays can intersect the magnetic axis and come into the region with the oppositely directed transverse plasma density gradient. Here they turn round and start deviate in the opposite direction. For most of the rays the final directions group near the "turning point". As the frequency increases, the turning point tends to move away from the magnetic axis, so that ultimately most of the rays leave the central invisible part of the emission cone and become observable. Obviously, this should result in some flattening or even a turn-up in the pulsar spectrum. In the present paper it is demonstrated that refraction of radio waves in pulsar plasma (along with the effects of observational geometry) really can account for the high-frequency features observed in the spectra of the core-dominated pulsars.

It should be pointed out that the high-frequency spectral breaks are peculiar not only to the core-dominated pulsars: They are often connected to the inversion in the intensity ratio of the conal components (for the examples and references see Sect. 4). Note that the theory of the electron-positron cascade above the polar cap of a pulsar allows, in principle, a non-axisymmetric distribution of the secondary plasma, which can underlie the difference in the intensities of the conal components at a fixed frequency (e.g. Arons 1993). At the same time, the distinction of the component spectra remains obscure. Indeed, continuity of the plasma flow in the tube implies that the plasma number density decreases as $r^{-3}$, independently of the azimuthal coordinate. Given the radius-to-frequency mapping, the radial decrease of the density is reflected in the frequency evolution of the intensity emitted. Hence, the inversion of the intensity ratio of the conal components is hardly attributable to the emission mechanism.
In the present paper we consider refraction in the plasma with a non-axisymmetric density distribution and suggest an explanation for the intensity ratio inversion on the basis of this phenomenon. Provided that the azimuthal density distribution has extremums, the rays are refracted towards the minima. This results in ray grouping in certain domains of the emission cone, the effect being more prominent at higher frequencies. (Note that such a patchy structure of the emission cone has an observational support, e.g. Lyne \& Manchester 1988). The original intensity is the highest in the regions of the plasma density maxima, whereas refraction acts to group the rays near the density minima. As refraction is frequency-dependent, the resultant structure of the emission beam can evolve significantly. Moreover, the emission patches move because of the tube shrinkage, so that for a fixed sight-line traverse the intensity ratio of the conal components can vary essentially. It will be shown below that the inversion in the intensity ratio of the conal components and the consequent spectral break can indeed be attributed to refraction in the plasma with a non-axisymmetric density distribution.

Within the frame of our explanation of the highfrequency features of pulsar spectra, the frequency of the spectral breaks, typically about $1 \mathrm{GHz}$, is interpreted as a characteristic frequency, at which refraction becomes sufficiently strong. It should be mentioned that for a number of pulsars the break in the spectrum almost coincides with the breaks in the frequency dependencies of the pulse width and linear polarization (McKinnon 1997). Both these dependencies can also be explained in terms of the propagation effects, with the break frequency having exactly the same physical meaning (Lyubarskii \& Petrova 1998; Petrova 2001).

The present paper continues the investigation of the observational consequences of refraction in pulsar magnetospheres. It is shown that refraction together with the effects of observational geometry can account for the highfrequency features in pulsar spectra. In Sect. 2 we write the ray equations for the case of an axisymmetric plasma density distribution. The behaviour of rays is discussed and the resultant spectra are presented. Refraction in the plasma with a non-axisymmetric density distribution is considered in Sect. 3. It is demonstrated that the effect can drive the frequency evolution of the component intensity ratio in a double profile and cause a break in the overall spectrum. The simulated spectra are discussed in Sect. 4. The results are summarized in Sect. 5 .

\section{The effect of refraction on the spectra of core-dominated pulsars}

Let us consider refraction in an ultrarelativistic electronpositron plasma embedded in a superstrong magnetic field of a dipolar structure. For this problem the assumptions concerning the magnetic field are reasonable. Firstly, the existence of non-dipolar components in the magnetosphere has no direct observational support (Arons 1993). Secondly, refraction is efficient only at distances of the 
order of the emission radius, i.e. sufficiently deep in the magnetosphere, so that rotational distortion of the magnetic field structure is insignificant. For the sake of clarity, the plasma is assumed to be cold in the proper rest frame. Although this is not the case in real pulsars, the results are qualitatively the same. Then the dispersion relation of the plasma takes the form (e.g. Lyubarskii 1995):

$\left(1-n^{2}\right)\left\{\left(1-n_{\|}^{2}\right)\left(1-\frac{\omega_{\mathrm{p}}^{2}}{\omega^{2} \gamma^{3}\left(1-n_{\|} \beta\right)^{2}}\right)-n_{\perp}^{2}\right\}=0$.

Here $n$ is the refractive index, $n_{\|}=c k_{\|} / \omega$ and $n_{\perp}=$ $c k_{\perp} / \omega, k_{\|}$and $k_{\perp}$ are the wave vector components parallel and perpendicular to the magnetic field, respectively, $\gamma$ is the plasma Lorentz-factor, $\beta$ the particle velocity in the units of $c, \omega_{\mathrm{p}}$ the plasma frequency,

$\omega_{\mathrm{p}}=\sqrt{\frac{4 \pi N e^{2}}{m}}$

where $N$ is the plasma number density, $e$ and $m$ are the electron charge and mass.

The ultrarelativistic highly magnetized plasma described by Eq. (1) allows three natural waves. The first parentheses correspond to the extraordinary wave. It has the vacuum dispersion law and therefore does not suffer refraction at all. (Note that the vacuum dispersion implies that direct generation of the extraordinary waves in the plasma is questionable). The expression in the curly brackets describes two ordinary waves. Given the oblique propagation with respect to the magnetic field, one of the ordinary waves is subluminous, while the other is superluminous. Although the subluminous waves can be easily generated (e.g. as a result of the two-stream instability in the plasma), they cannot leave the magnetosphere because of Landau damping. Therefore we are interested in refraction of the ordinary superluminous waves.

Direct generation of these waves in any conceivable plasma process seems to be impossible. However, they can arise as a result of induced scattering of the subluminous waves off the plasma particles. At the conditions relevant to pulsar plasma the scattering appears to be rather efficient (Lyubarskii 1996). The resultant superluminous waves acquire the frequencies of the order of the local Lorentz-shifted proper plasma frequency,

$\omega \sim \omega_{\mathrm{p}} \sqrt{\gamma}$

Despite the recent progress in the understanding of the physics of the polar gap, the distribution of the number density of the secondary plasma is not obtained yet. Therefore, we apply to the qualitative considerations. The lack of plasma at the centre of the open field line tube and beyond its boundaries suggests some decrease of the plasma number density towards the magnetic axis and tube edges. At the same time, continuity of the plasma flow within the tube results in radial number density decrease $\propto r^{-3}$ along any field line. Let us choose the polar coordinate system $(r, \chi)$ whose axis coincides with the magnetic axis. Then the dipolar field line is given by the equation: $\chi=\chi_{0} \sqrt{r / r_{0}}$. Keeping in mind all this, we take the plasma density distribution in the form of the two symmetrical Gaussians centered at the characteristic field lines $\left(\chi= \pm \chi_{\mathrm{c}}\right.$ at $\left.r=r_{0}\right)$ :

$N=N_{0}\left(\frac{r_{0}}{r}\right)^{3} \exp \left\{-\varepsilon\left(\frac{|\chi|-\chi_{\mathrm{c}} \sqrt{r / r_{0}}}{\chi_{\mathrm{c}} \sqrt{r / r_{0}}}\right)^{2}\right\}$.

Here $\varepsilon$ is a constant of order unity. The density distribution is axisymmetric, i.e. it is the same in each crosssection containing the magnetic axis. Thus, the "hollow tube" is assumed. Using Eqs. (2) and (4) in Eq. (3), one can see that a sort of radius-to-frequency mapping is the case: along a fixed field line $\omega \propto r^{-3 / 2}$.

In the problem considered all the scale lengths are much greater than the wavelength, so that the geometrical optics approximation is appropriate. Then the ray propagation is described by the Hamilton equations, which can be reduced to the form (Eq. (9) in Petrova 2000):

$r \frac{\mathrm{d} \chi}{\mathrm{d} r}=\frac{\chi}{2}+\frac{3(1+\eta)^{3}}{2 A}(\theta-\chi)$
$\frac{\mathrm{d} \theta}{\mathrm{d} r}=\frac{6(1-\eta) N(\theta-\chi)}{A r f_{0}^{2}}+\frac{8 \varepsilon(1+\eta) \eta N\left[\chi \chi_{0} /\left(\chi_{\mathrm{c}} \sqrt{r}\right)-1\right]}{3 A r^{3 / 2} f_{0}^{2} \chi_{0} \chi_{\mathrm{c}} \gamma^{2}}$
$\eta\left(1-\frac{4 N}{f_{0}^{2} r^{3}(1+\eta)^{2}}\right)-\frac{9}{4} \chi_{0}^{2} \gamma^{2}(\theta-\chi)^{2}=0$

where

$A \equiv(1+\eta)^{3}-\frac{4(1-\eta) N}{f_{0}^{2}}, \quad \eta \equiv 2 \gamma^{2}\left(1-n_{\|}\right)$

and the quantities $r, \chi, \theta, N$ are normalized by their initial values; $\theta$ is the wavevector tilt to the magnetic axis, $f \equiv$ $\omega /\left(\omega_{\mathrm{p}} \sqrt{\gamma}\right)$ and the subscript " 0 " refers to the quantities at the initial point. The rays are supposed to be emitted along the magnetic lines, so that $\theta_{0}=3 \chi_{0} / 2$.

The set of Eq. (5) describes the ray trajectory and the wavevector evolution in the course of refraction. The wavevector deviation is determined by the variation of the refractive index along the trajectory. In our case this variation is due to the magnetic line curvature (the first term in the right-hand side of the second equation in the set (5)) and also due to the variation of the plasma number density (the second term). Note that as the open field line tube is narrow, the transverse scale length is much less than the radial one, so that the transverse density gradient is much more significant. Its contribution is $\sim\left(\chi_{0} \chi_{\mathrm{c}}\right)^{-1} \gamma^{-2}$. Therefore refraction is more efficient for the rays emitted closer to the magnetic axis (at smaller $\chi_{0}$ ) and at lower altitudes (for a fixed characteristic field line, smaller $\chi_{\mathrm{c}}$ imply smaller emission altitudes $r_{0}$ ). It should be noted that the equations of the set (5) do not depend on $r_{0}$ explicitly. Given that $f_{0}$ is a constant of order unity, only $\chi_{\mathrm{c}}$ is related to the frequency of the ray: $\chi_{\mathrm{c}} \propto r_{0}^{1 / 2} \propto \nu^{-1 / 3}$.

At high enough frequencies it is the transverse density gradient that determines refraction. Ray behaviour is qualitatively dependent on the sign of this gradient. Let us consider the inner rays (i.e. the rays emitted in the region 
$\left.|\chi|<\chi_{\mathrm{c}}\right)$. From the second equation of (5) it is clear that they start deviate towards the magnetic axis (for more details concerning ray trajectories and wavevector evolution see Petrova 2000). Figure 1 shows the numerically calculated final wavevector tilts as functions of the initial one for the three values of $\chi_{\mathrm{c}}\left(\chi_{\mathrm{c}}=0.04,0.03\right.$ and 0.02), i.e. for the three frequencies related to each other as 1:2.4:8. The initial tilts are equidistant, so that visible nonuniformity in the distribution of the labels along the curves testifies to the concentration of rays on account of refraction. The dashed lines bound the region in the central part of the emission cone, which is invisible because of the non-central sight-line trajectory. One can see that refraction tends to place the rays into the invisible region, the effect becoming more prominent at higher frequencies. For the rays emitted at smaller $\chi_{0}$ refraction is particularly strong. It makes them come into the opposite half of the tube, turn round and start deviate in the opposite direction. As is evident from Fig. 1, at higher frequencies the turning point of the rays shifts outwards, so that ultimately a great deal of rays can become visible.

Thus, refraction leads to a significant redistribution of rays within the emission beam. The final intensity profile and spectrum are determined by both the initial angular intensity distribution and the ray grouping because of refraction. Here we consider a uniform distribution of the intensity within the hollow cone,

$$
I\left(\theta_{0}\right)=1, \quad \frac{3}{4} \chi_{\mathrm{c}} \leq\left|\theta_{0}\right| \leq \frac{3}{2} \chi_{\mathrm{c}},
$$

and the Gaussian distribution similar to that of the plasma number density:

$$
\begin{aligned}
& I\left(\theta_{0}\right)=\exp \left[-\zeta \varepsilon\left(\frac{2\left|\theta_{0}\right| / 3-\chi_{\mathrm{c}}}{\chi_{\mathrm{c}}}\right)^{2}\right], \\
& \frac{3}{4} \chi_{\mathrm{c}} \leq\left|\theta_{0}\right| \leq \frac{3}{2} \chi_{\mathrm{c}},
\end{aligned}
$$

with $\zeta$ being a constant of order unity. Energy conservation along the phase trajectories of the rays implies that in any axial cross-section of the open field line tube

$$
I_{r_{0}}\left(\theta_{0}\right) \mathrm{d} \theta_{0}=I_{r}(\theta) \mathrm{d} \theta
$$

where $I_{r}(\theta)$ is the angular distribution of the intensity at the distance $r$. Using Eqs. (5) in (8) one can find the final intensity distribution. The influence of refraction on the morphology of pulse profiles is studied in Petrova (2000).

The spectra resulting from the numerical calculations on the basis of Eqs. (5)-(8) for the observational geometry specified in Fig. 1 are presented in Fig. 2. The dashed line shows the original spectral slope. According to the theory of induced scattering (Lyubarskii 1996), it is taken to be $-4 / 3$. One can see that taking into account refraction together with the effects of observational geometry can modify the original spectrum significantly. As refraction becomes more efficient at higher frequencies, the increasingly strong grouping of the rays in the central invisible

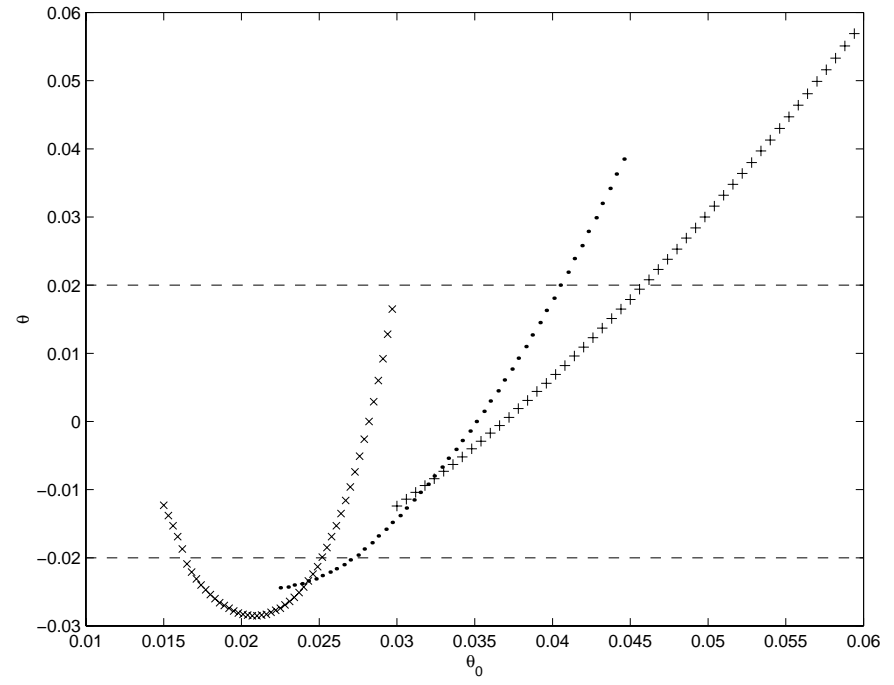

Fig. 1. The influence of refraction on the wavevector orientation in case of the axisymmetric plasma density distribution. The final wavevector tilt, $\theta$, is plotted as a function of the initial one, $\theta_{0}$, for the various values of $\chi_{\mathrm{c}}: \chi_{\mathrm{c}}=0.02(\times), 0.03(\cdot)$ and $0.04(+) ; \gamma=30, f_{0}=0.5, \varepsilon=3, \theta_{0} \leq 3 \chi_{\mathrm{c}} / 2$. The dashed lines bound the range, which is invisible in the case of non-central sight-line trajectory across the emission beam (the impact angle is assumed to be 0.02).

part of the emission cone leads to a break in the resultant spectrum. At still higher frequencies, when increasingly great number of rays leave the invisible region, the spectrum suffers flattening. These features appear to be more prominent for the uniform distribution of the intensity emitted, however, they are still observable for the Gaussian distribution with $\zeta=2$. The quantitative results presented in Fig. 2 depend on the chosen plasma density distribution and the distribution of the plasma particles in energy. Hence, these spectra only illustrate the qualitative features introduced by refraction given the appropriate plasma parameters. Slight changes in the assumed plasma parameters as well as the original intensity distribution are expected to result in some variation of the final spectral slopes. In particular, the flattening of the spectrum may grow into a turn-up. Note that a certain diversity in the observed pulsar spectra testifies to an actual diversity in the parameters of the magnetospheric plasma.

In our calculations the frequency of the spectral break, $\nu_{\mathrm{c}}$, corresponds to the emission altitude, $r_{0}$, at which the polar angle of the characteristic field line $\chi_{\mathrm{c}}=0.06$. The value of $\nu_{\mathrm{c}}$ can be find as follows. It is convenient to normalize the plasma number density at the characteristic field line by the Goldreich-Julian charge density:

$N=\frac{\kappa B}{e c P}$

where $\kappa$ is the multiplicity factor of the secondary plasma at this field line, $B$ the strength of the ambient magnetic field, $P$ the pulsar period. The characteristic field line lies within the open field line tube, so that $u \equiv \chi_{\mathrm{c}} / \chi_{\mathrm{t}} \lesssim 1$; here $\chi_{\mathrm{t}} \equiv \sqrt{r_{0} / r_{\mathrm{L}}}$ is the polar angle of the last open field 
line at $r_{0}, r_{\mathrm{L}} \equiv 5 \times 10^{9} \mathrm{P} \mathrm{cm}$ is the light cylinder radius. Using Eqs. (2) and (3) and recalling that $B \propto r^{-3}$, we find:

$$
\begin{aligned}
\nu_{\mathrm{c}}= & P^{-2} u^{3}\left(\frac{f_{0}}{0.5}\right)\left(\frac{r_{\star}}{10^{6} \mathrm{~cm}}\right)^{3 / 2} \\
& \times\left(\frac{\kappa}{10^{4}} \cdot \frac{\gamma}{30} \cdot \frac{B_{\star}}{10^{12} \mathrm{G}}\right)^{1 / 2} \mathrm{GHz},
\end{aligned}
$$

where the subscript "*" refers to the values at the neutron star surface.

As follows from Eq. (10), at typical values of the parameters, $\nu_{\mathrm{c}} \sim 1 \mathrm{GHz}$. One can see that the explicit period dependence of $\nu_{\mathrm{c}}$ is rather strong, however, the other quantities in Eq. (10) (except $r_{\star}$ and $B_{\star}$ ) can depend on the period implicitly. It should be pointed out that the observed dependence, $\nu_{\mathrm{c}} \propto P^{-1 / 2}$ (Malofeev \& Malov 1980; Malov \& Malofeev 1991), can be obtained, if one assumes that the location of the characteristic field line is independent of the tube width, i.e. $u \propto P^{1 / 2}$. At present it is difficult to conclude whether this assumption is reasonable. Note that the pulsar period can affect the development of the electron-positron cascade, so that the quantities $\kappa$ and $\gamma$ can also be period-dependent.

One more issue should be noted here. Within the scope of the present consideration, the core-dominated pulsars are those, in which most part of the emission originates in the region of the inward plasma density decrease (i.e. at $\left.|\chi|<\chi_{\mathrm{c}}\right)$. As the observed profile shape can be strongly influenced by refraction, this definition may differ from the customary one, which is based on the observed profile morphology.

\section{The influence of refraction on the spectral behaviour of conal components}

Now let us turn to the problem of refraction in the case of a non-axisymmetric distribution of the magnetospheric plasma. The theory of pair creation above the polar cap of a pulsar does not exclude such a distribution (e.g. Arons 1993). For the sake of clarity we take the following distribution law for the plasma number density within the open field line tube:

$$
\begin{aligned}
N= & N_{0}\left(\frac{r_{0}}{r}\right)^{3} \exp \left\{-\varepsilon\left(\frac{|\chi|-\chi_{\mathrm{c}} \sqrt{r / r_{0}}}{\chi_{\mathrm{c}} \sqrt{r / r_{0}}}\right)^{2}\right\} \\
& \times \exp \left[\mu \cos ^{2}\left(\psi+\psi_{\mathrm{c}}\right)\right],
\end{aligned}
$$

with

$\mu= \begin{cases}1, & -\pi / 2 \leq \psi+\psi_{\mathrm{c}} \leq \pi / 2 \\ 0, & \pi / 2<\psi+\psi_{\mathrm{c}}<3 \pi / 2\end{cases}$

Here the spherical coordinate system $(r, \chi, \psi)$ is chosen; its polar axis coincides with the magnetic axis, while the meridional plane at $\psi=0$ contains the rotational axis of a pulsar. Equations (11) and (12) imply that in one half of the tube the plasma density distribution coincides with that considered in the previous section (cf. Eq. (4)), whereas in the other half the azimuthal density distribution has a maximum, which is shifted from the fiducial plane by $\psi_{\mathrm{c}}$. One can see that along any fixed field line the number density decreases as $r^{-3}$, so that the continuity condition is satisfied. Let the wavevector direction of the outgoing rays be characterized by the polar and azimuthal angles, $\theta$ and $\phi$. Then the rays are detectable by an observer on condition that (Manchester \& Taylor 1977):

$\tan \phi= \pm \sqrt{\frac{\theta^{2}}{(\alpha-\xi)^{2}}-1}$

where $\alpha$ is the angle between the magnetic and rotational axes, $\xi$ the wavevector tilt to the rotational axis.

Given the non-axisymmetric plasma density distribution (11)-(12), the ray equations take the form (cf. Eqs. (7) and (17) in Petrova \& Lyubarskii 2000):

$$
\begin{aligned}
& r \frac{\mathrm{d} \chi}{\mathrm{d} r}=\frac{\chi}{2}+\frac{3(1+\eta)^{3}}{2 A}[\theta \cos (\phi-\psi)-\chi] \\
& r \chi \frac{\mathrm{d} \psi}{\mathrm{d} r}=\frac{3(1+\eta)^{3} \theta}{2 A} \sin (\phi-\psi) \\
& \frac{\mathrm{d} \theta}{\mathrm{d} r}=\frac{6(1-\eta) N}{A r f_{0}^{2}}[\theta-\chi \cos (\phi-\psi)]- \\
& \frac{8 \varepsilon(1+\eta) \eta N\left(\chi \chi_{0} /\left(\chi_{\mathrm{c}} \sqrt{r}\right)-1\right) \cos (\psi-\phi)}{3 A r^{3 / 2} f_{0}^{2} \chi_{0} \chi_{\mathrm{c}} \gamma^{2}}- \\
& \frac{8 \mu \varepsilon(1+\eta) \eta N \sin (\psi-\phi) \sin \left(\psi+\psi_{\mathrm{c}}\right) \cos \left(\psi+\psi_{\mathrm{c}}\right)}{3 A r f_{0}^{2} \chi_{0}^{2} \chi \gamma^{2}} \\
& \theta \frac{\mathrm{d} \phi}{\mathrm{d} r}=\frac{6(1-\eta) N \chi}{A r f_{0}^{2}} \sin (\phi-\psi)- \\
& \frac{8 \varepsilon(1+\eta) \eta N\left(\chi \chi_{0} /\left(\chi_{\mathrm{c}} \sqrt{r}\right)-1\right) \sin (\phi-\psi)}{3 A r^{3 / 2} f_{0}^{2} \chi_{0} \chi_{\mathrm{c}} \gamma^{2}}+ \\
& \frac{8 \mu \varepsilon(1+\eta) \eta N \cos (\psi-\phi) \sin \left(\psi+\psi_{\mathrm{c}}\right) \cos \left(\psi+\psi_{\mathrm{c}}\right)}{3 A r f_{0}^{2} \chi_{0}^{2} \chi \gamma^{2}} \\
& \eta\left[1-\frac{4 N}{f_{0}^{2} r^{3}(1+\eta)^{2}}\right]-\frac{9}{4} \chi_{0}^{2} \gamma^{2} \\
& \times\left[\chi^{2}+\theta^{2}-2 \chi \theta \cos (\phi-\psi)\right]=0 .
\end{aligned}
$$

Here the quantities $r, \chi, \theta$ and $N$ are normalized by their initial values; $A$ and $\eta$ are defined after Eq. (5). As the rays are emitted along the magnetic lines, $\theta_{0}=3 \chi_{0} / 2$ and $\phi_{0}=\psi_{0}$. In the absence of the azimuthal density gradient, i.e. if $\mu \equiv 0$, Eq. (14) certainly turns into Eq. (5). Indeed, in this case $\psi \equiv \phi \equiv$ const. The azimuthal density gradient makes the wavevector deviate from the initial plane of the magnetic lines (see the third addend in the next to the last equation of the set (14)). The consequent difference between $\psi$ and $\phi$ implies that the trajectory goes off the initial plane as well (see the second equation in Eq. (14)). The wavevector and trajectory both deviate in the same direction, oppositely to the azimuthal density gradient, i.e. in the direction of the plasma density decrease. Refraction again appears to be more efficient for the rays emitted at smaller polar angles and at lower altitudes (i.e. at higher frequencies). 


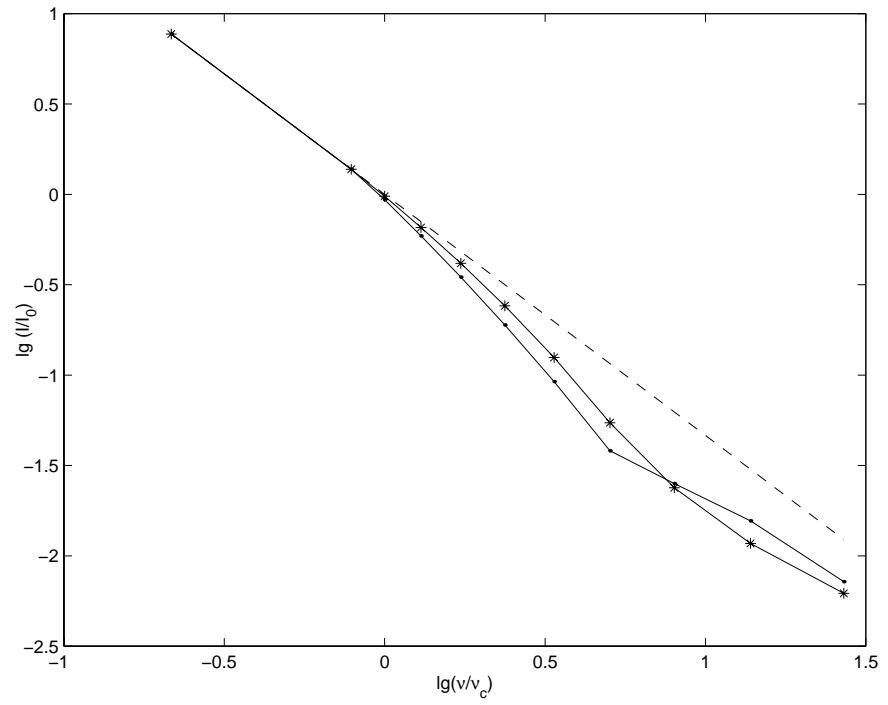

Fig. 2. The core-component spectra calculated accounting for refraction. The parameters are the same as in Fig. 1 and the intensity is normalized arbitrarily. The original intensity distribution is given by Eq. (6) (points) and Eq. (7) at $\zeta=2$ (asterisks). The dashed line shows the original spectral slope $-4 / 3$.

Figure 3 demonstrates the final structure of the emission beam at the two frequencies, that are related to each other as 1:4.6 (Figs. 3a and 3b, respectively). The final wavevector tilts are calculated numerically on the basis of Eq. (14). In each of the figures the rays are emitted at three polar angles, $\chi=\chi_{\mathrm{c}}, 1.25 \chi_{\mathrm{c}}$ and $1.5 \chi_{\mathrm{c}}$, and they are initially equidistant in the azimuthal coordinate. One can observe that refraction leads to redistribution of the rays in azimuth: they tend to concentrate away from the density maximum. In Fig. 3a the effect is barely distinguishable, while in Fig. $3 \mathrm{~b}$ it is visible clearly. Thus, ray grouping becomes more prominent at higher frequencies.

As the density maximum is shifted from the fiducial meridional plane $\psi=0$, the concentration of rays on both edges of the sight-line trajectory is different, especially at higher frequencies. This should reveal itself in the distinction of the intensities of the resultant conal components. Note that the component, which is more distant from the density maximum, is enhanced, while the other weakens. At the same time, the original intensity is expected to be higher close to the plasma density maximum. Hence, refraction acts to reverse the intensity ratio of the components.

To study the spectral behaviour of the conal components in more detail it is necessary to trace the evolution of the intensity distribution in the emission beam on account refraction. The original intensity distribution is taken in the form

$$
\begin{aligned}
& I\left(\theta_{0}, \phi_{0}\right)=\exp \left[-\zeta \varepsilon\left(\frac{2\left|\theta_{0}\right| / 3-\chi_{\mathrm{c}}}{\chi_{\mathrm{c}}}\right)^{2}\right] \\
& \times \exp \left[\mu \cos ^{2}\left(\phi_{0}+\psi_{\mathrm{c}}\right)\right], \quad \frac{3}{2} \chi_{\mathrm{c}} \leq\left|\theta_{0}\right| \leq \frac{9}{4} \chi_{\mathrm{c}}
\end{aligned}
$$
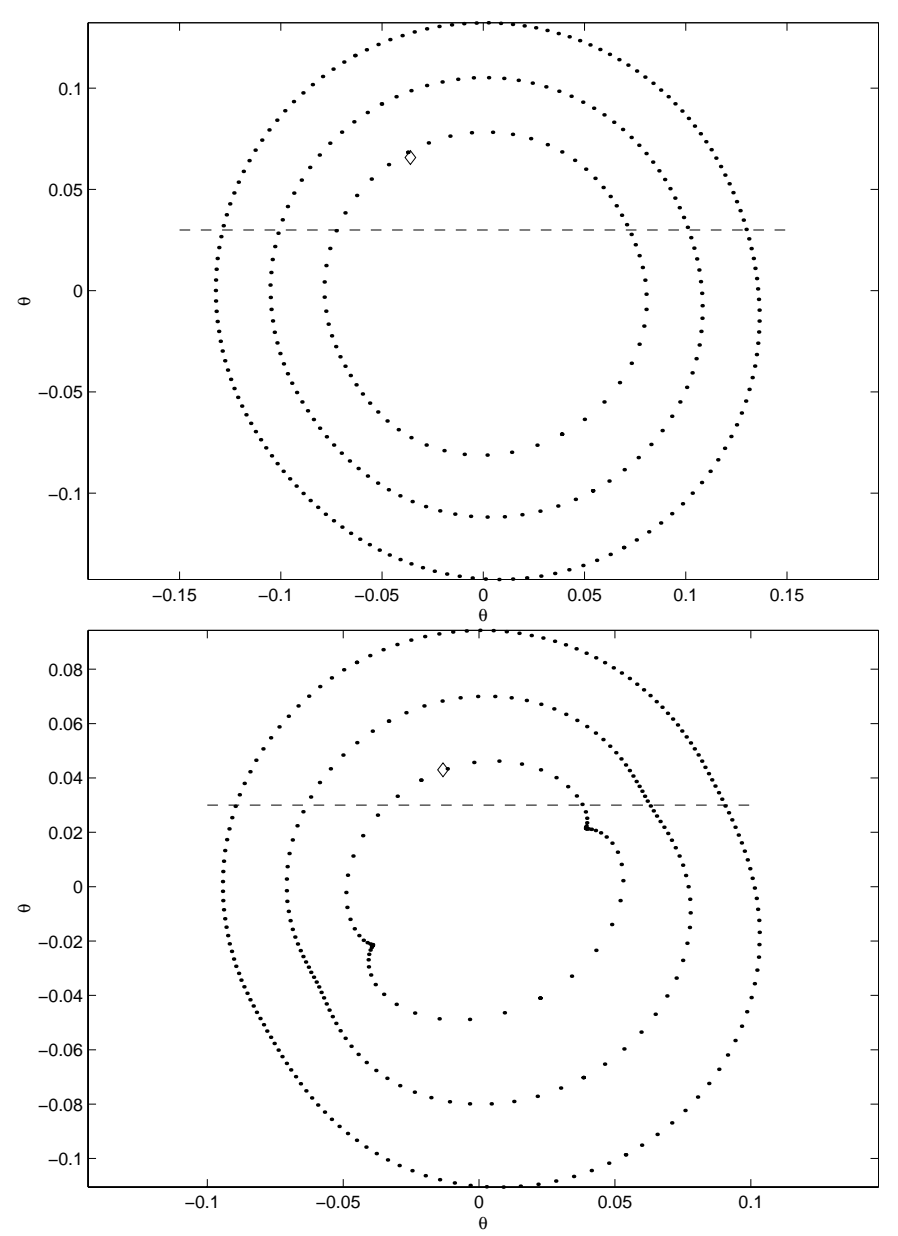

Fig. 3. Redistribution of rays in azimuth as a result of refraction in the plasma with the non-axisymmetric density distribution (11): a) $\left.\chi_{\mathrm{c}}=0.05, \mathbf{b}\right) \chi_{\mathrm{c}}=0.03 ; \gamma=30, f_{0}=0.5, \varepsilon=1$, $\chi_{0}=\chi_{\mathrm{c}}, 1.25 \chi_{\mathrm{c}}$ and $1.5 \chi_{\mathrm{c}}$. The diamond denotes the location of the maximum of the plasma density $\left(\theta=3 \chi_{\mathrm{c}} / 2, \phi=-\psi_{\mathrm{c}}\right)$. An arbitrary sight-line trajectory is shown by the dashed line.

which is similar to the plasma density distribution (11). Owing to refraction the trajectories of the rays in the phase space $(r, \theta, \phi)$ can diverge or converge as $r$ increases. Since the energy is transferred along the phase trajectories of the rays, for any initial point $\left(r_{0}, \theta_{0}, \phi_{0}\right)$ the evolution of the intensity $I_{r_{0}}\left(\theta_{0}, \phi_{0}\right)$ with $r$ is determined by the deformation of the elementary area $\theta_{0} \mathrm{~d} \theta_{0} \mathrm{~d} \phi_{0}$, that is bounded by the phase trajectories of the nearby rays:

$I_{r_{0}}\left(\theta_{0}, \phi_{0}\right) \theta_{0} \mathrm{~d} \theta_{0} \mathrm{~d} \phi_{0}=I_{r}(\theta, \phi) \theta \mathrm{d} \theta \mathrm{d} \phi$.

Hence,

$I_{r}(\theta, \phi)=I_{r_{0}}\left(\theta_{0}, \phi_{0}\right) \frac{\theta_{0}}{\theta}\left[\frac{\partial(\theta, \phi)}{\partial\left(\theta_{0}, \phi_{0}\right)}\right]^{-1}$,

where

$\frac{\partial(\theta, \phi)}{\partial\left(\theta_{0}, \phi_{0}\right)} \equiv \frac{\partial \theta}{\partial \theta_{0}} \cdot \frac{\partial \phi}{\partial \phi_{0}}-\frac{\partial \theta}{\partial \phi_{0}} \cdot \frac{\partial \phi}{\partial \theta_{0}}$

is the Jacobian describing the transition from the coordinates $\left(\theta_{0}, \phi_{0}\right)$ to the coordinates $(\theta, \phi)$. It can be found through numerical calculation of the ray tracings on the 
basis of Eq. (14). Then from Eq. (17) we can obtain the final intensity distribution in the emission beam, $I_{\mathrm{f}}(\theta, \phi)$. The total intensity detected by an observer is given by the integral

$\int I_{\mathrm{f}}(\theta, \phi) \mathrm{d} \theta$

which is taken over the sight-line trajectory across the emission beam.

The spectra calculated on the basis of Eqs. (14)-(19) for the viewing geometry shown in Fig. 3 are plotted in Fig. 4. One can see that at the frequencies $\sim \nu_{\mathrm{c}}$ the intensity ratio of the components is reversed. At the lower frequencies the component intensities are mainly determined by the original intensity distribution. So the component, which is located closer to the density maximum (points), is stronger. At the higher frequencies the intensity distribution appears to be essentially affected by refraction. As the sight-line trajectory passes through the region of ray concentration, the second component (pluses) is considerably enhanced in intensity and becomes the dominant. This is accompanied by a significant flattening of the component spectrum (see Fig. 4). At the same time, the spectrum of the first component is steeper than the original one. Indeed, the lack of rays close to the plasma density maximum becomes more prominent with the frequency. At still higher frequencies the sight-line trajectory shifts away from the region of ray concentration because of the shrinkage of the emission cone. Then both intensities rapidly decrease (while the amplitude ratio remains the same) causing the break in the total spectrum. Thus, the inversion of the intensity ratio of the conal components and the break in the overall spectrum can both be explained by refraction in the plasma with a non-axisymmetric distribution.

The results obtained in the present section concern not only the double profiles. They are also applicable to the outer conal components (i.e. those emitted at $\chi>\chi_{\mathrm{c}}$ ) of the more complex profiles. Then the total spectrum is the sum of those shown in Figs. 2 and 4. Note that the non-axisymmetry in the plasma density distribution can affect the core components as well. It is expected to result in an asymmetry of the core component with respect to the profile centre. As the effect is usually superposed on the geometrical effect discussed in the previous section, it is difficult to generalize the features of the resultant core spectra in this case.

\section{Discussion}

Refraction of radio waves in pulsar plasma causes a marked redistribution of the intensity in the emission beam. Then for a given sight-line trajectory the integral intensity of the profile can be changed considerably, though the total energy of the emission beam certainly remains the same. As refraction is frequency-dependent, it affects the pulsar spectrum. Above it has been demonstrated that the spectral changes can be peculiar to both the core and conal components.

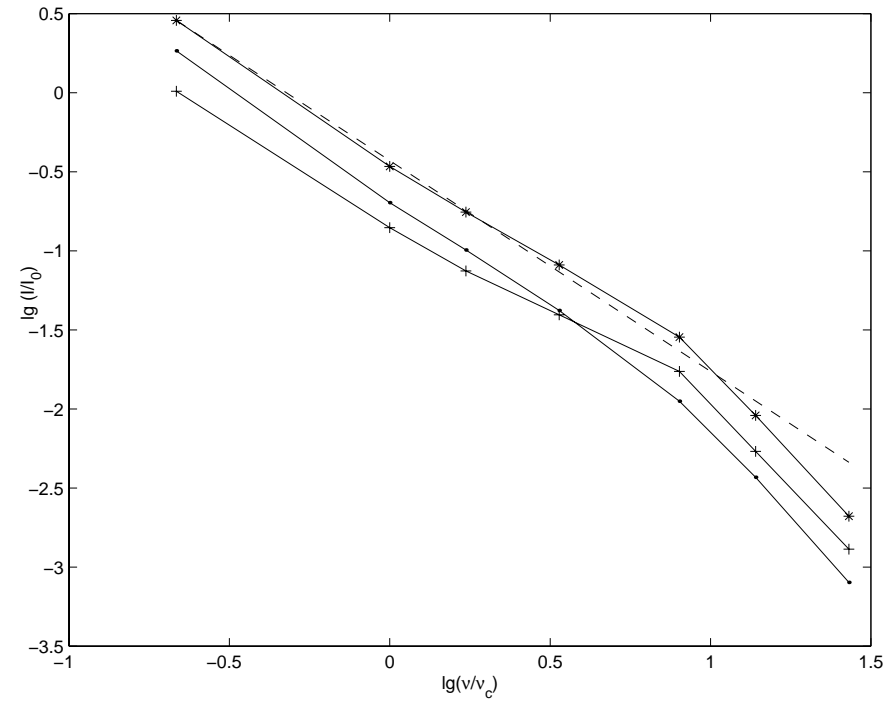

Fig. 4. The spectra calculated accounting for refraction in the plasma with the density distribution (11). The parameters are the same as in Fig. 3; + and - - the conal component spectra, * - the overall spectrum. The dashed line shows the original spectral slope $-4 / 3$.

The observational data allow us to divide the pulsars with the spectral breaks into several groups according to the frequency behaviour of the profile shape (the comprehensive data on pulsar spectra are presented in Malofeev et al. 1994, Malofeev 1999; the corresponding profiles at different frequencies can be found in Kuzmin \& Losovskii 1999 (100 MHz), Lyne \& Manchester 1988 (up to $1.4 \mathrm{GHz}$ ), Seiradakis et al. 1995 (1.4, 4.75 and $10.55 \mathrm{GHz}$ ) and von Hoensbroech 1999 (5 GHz)). The first group contains the core-single pulsars (e.g. PSR $0823+26$, PSR 1706-16 and PSR 1839+56) with the persistent morphology over a wide frequency range. The pulsars PSR 1929+10 and PSR 2021+51, which exhibit a turn-up at extremely high frequencies (Kramer et al. 1996), also seem to enter into this group. The second group is represented by the triple pulsars with a significant change of the amplitude ratio of the core and conal components with the frequency (e.g. PSR 2045-16 and PSR 2111+46). Here one should also consider the double-profile pulsars, which are classified as core $+1 / 2$ cone according to the polarization data (e.g. PSR $0355+54$ and PSR 0450+55); these profiles also show a marked evolution of the intensity ratio of the components. For the two above mentioned groups of pulsars the spectral break is likely to occur due to considerable steepening of the core-component spectrum at high frequencies, so that their spectra can be modelled by Fig. 2 of the present paper.

The next group is composed of the double-conal pulsars with a marked frequency evolution of the component intensity ratio (e.g. PSR 0148-06, PSR 0525+21, PSR $0834+06$ and PSR $1133+16)$. Their spectral evolution is similar to that simulated in Sect. 3 (see Fig. 4). The last group consists of the pulsars, in which both the steepening of the core-component spectrum and the 
inversion of the intensity ratio of the conal components seem to contribute essentially (e.g. PSR 0450-18, PSR $1237+25$, PSR 1642-03 and PSR 1821+05). Then the overall spectrum is believed to be the sum of the component spectra plotted in Figs. 2 and 4. Thus, the features of the pulsar spectra are coupled to the profile morphology and its frequency evolution. Within the frame of our model this relation is explained naturally: both the spectrum and the profile shape are affected by refraction.

Note that the main features of the observed frequency evolution of the pulse width (Rankin 1983) can also be attributed to refraction (Lyubarskii \& Petrova 1998). At low frequencies the pulse width, $w$, is determined by the width of the open field line tube and therefore decreases with the wavelength, whereas at high frequencies it is affected by refraction and remains nearly constant. As a rule, such a dependence $w(\nu)$ is characteristic of the double-conal pulsars (e.g. PSR $1133+16$ and PSR $0525+21$ ). The frequency of the break in $w(\nu)$ often coincides with that in the total-intensity spectrum (McKinnon 1997), both effects being the consequences of refraction. Some pulsars exhibit quite another dependence $w(\nu)$ : At the frequencies $\sim 100 \mathrm{MHz}-1 \mathrm{GHz}$ the power law is damaged by the so-called "absorption feature" (Rankin 1983). Such a behaviour of the pulse width is usually met in the core components (e.g. PSR 1642-03, PSR 1706-16 and PSR 1933+16). The "deficit" of pulse width can also be explained by the peculiarities in the behaviour of the core rays on account of refraction (Lyubarskii \& Petrova 1998), i.e. exactly in the same manner as the "deficit" in the total intensity of the core components (see Sect. 2). However, these dependencies bear only qualitative resemblance. The observations show that the "absorption features" in $w(\nu)$ and in the spectrum are considerably shifted in frequency. The point is that the observed pulse width is determined by the final intensity in the profile (it is measured at the one-half of the maximum intensity). Hence, strictly speaking, it depends on the original intensity distribution and ray grouping, rather than by the behaviour of a chosen "boundary" ray. For the same reason there are some counter-examples of the relation between the profile morphology and the form of $w(\nu)$.

One more observational consequence of refraction concerns the degree of linear polarization in the observed pulsar radiation. It has been already shown (Petrova 2001) that refraction can cause the quasi-longitudinal propagation of the waves with respect to the magnetic field. Then the ordinary superluminous waves are partially converted into the extraordinary ones. The resultant superposition of the orthogonally polarized modes is believed to underlie the high-frequency depolarization of pulsar radiation. The frequency dependence of linear polarization usually has the break, which accords with the breaks in the totalintensity spectrum and $w(\nu)$ (McKinnon 1997).

The properties of the observed radiation at the low and high frequencies appear to be somewhat different (Xilouris et al. 1996), though the emission mechanism is believed to be the same. One can expect that at high enough frequen- cies $\left(>\nu_{\mathrm{c}}\right)$, where refraction is significant, it is the plasma density distribution that determines the observed characteristics of pulsar radiation. The observational data testify to the correlation of the profile morphology, the spectral index and the degree of linear polarization with the accelerating voltage in the polar gap (von Hoensbroech 1999). The older and less energetic pulsars usually have the double conal profiles, the steeper spectra and low polarization. The core components are peculiar to young highly energetic pulsars; the conal outriders in the triple profiles are characterized by much flatter spectra and high polarization. Hence, one can conclude that the process of pair creation and the consequent distribution of the secondary plasma depend considerably on the pulsar parameters (in particular, on the period and period derivative). Note that the correlation of the characteristics of pulsar radiation with the accelerating potential is prominent only at the high enough frequency, $\nu=4.9 \mathrm{GHz}$; at $\nu=1.4 \mathrm{GHz}$ it is weak, while at $\nu=0.4 \mathrm{GHz}$ it is absent at all. The point is that at low frequencies, $\nu<\nu_{\mathrm{c}}$, the characteristics of pulsar radiation are mainly determined by the radio emission process rather than by refraction. Although in this case they also can depend on the pulsar parameters, this dependence may be not so straightforward.

It is interesting to note that at $\nu \lesssim \nu_{\mathrm{c}}$ the profile components are usually comparable in intensity. As is demonstrated in the present paper, they can have different spectra because of refraction, and therefore at extremely high frequencies, $\nu \gg \nu_{\mathrm{c}}$, only the component with the flattest spectrum can be detectable. The observations show that at $\nu \sim 10-30 \mathrm{GHz}$ the profiles indeed have a simple form (e.g. Xilouris et al. 1996).

\section{Conclusions}

We have considered refraction of the ordinary superluminous waves in an ultrarelativistic highly magnetized plasma of pulsars. The effect appears to be determined by the plasma number density gradient across the open field line tube. Both the wavevector and ray trajectory tend to deviate in the direction of the plasma density decrease. Strong enough refraction causes the grouping of the final wavevector orientations in those parts of the emission beam, which are projected to the minima of the plasma number density. The higher the frequency - the stronger refraction. It is found that for the typical parameters of pulsar plasma refraction leads to a significant redistribution of the intensity in the emission beam at the frequencies above $1 \mathrm{GHz}$. This should affect the observed pulsar spectrum.

It is demonstrated that refraction acts to place the core rays (i.e. those emitted at $\theta_{0}<3=\chi_{\mathrm{c}} / 2$ ) into the central part of the emission beam, which is invisible given the non-central sight-line trajectory, so that the spectrum of the core component steepens. At still higher frequencies the rays can leave the invisible region and the spectrum flattens. Then the intensity tends to the values corresponding to the extrapolation of the original 
low-frequency spectrum. Thus, the high-frequency spectral features of the core components can be attributed to refraction. Furthermore, refraction in the plasma with a non-axisymmetric density distribution can influence the spectral behaviour of the outer conal components. Provided that the azimuthal density distribution has an extremum, which is shifted from the central plane containing the magnetic and rotational axes, refraction can change the spectra of the conal components in a different manner. The spectrum of the component, that originates in the region of the higher number density, becomes steeper, whereas the spectrum of another component flattens. Given that the higher number density entails the higher original intensity, the ratio of the component intensities can reverse with the frequency. This is followed by the break in the overall spectrum of the double-conal pulsar.

The observational consequences of refraction in the magnetospheric plasma concern not only the pulsar spectra. Refraction can also modify the pulse width and cause the high-frequency depolarization in the observed radiation (Lyubarskii \& Petrova 1998; Petrova 2001). The spectral break often concurs with the breaks in the frequency dependencies of the pulse width and linear polarization (McKinnon 1997), indicating that the underlying phenomenon is the same. Within the frame of these views one can conclude that at the low enough frequencies, $<1 \mathrm{GHz}$, the characteristics of pulsar radiation are mainly determined by the radio emission mechanism, while at the higher frequencies refraction plays the dominant role. Hence, the high-frequency properties of pulsar radiation reflect the distribution of the plasma number density across the open field line tube. This is confirmed by the observed correlation of the profile morphology, the spectral index and the degree of linear polarization at high frequencies with the accelerating voltage in the polar gap (von Hoensbroech 1999).

\section{References}

Arons, J. J. 1993, ApJ, 408, 160

Arons, J. J., \& Scharlemann, E. T. 1979, ApJ, 231, 854

Kramer, M., Xilouris, K., Jessner, A., et al. 1996, A\&A, 306, 867

Kuz'min, A. D., \& Losovskii, B. Ya. 1999, AZh, 76, 338 [English translation: Astron. Rep., 76, 228]

Lyne, A. G., \& Manchester, R. N. 1988, MNRAS, 234, 477

Lyubarskii, Yu. E. 1995, Astrophys. Space Phys. Rev., 9, pt. 2,1

Lyubarskii, Yu. E. 1996, A\&A, 308, 809

Lyubarskii, Yu. E., \& Petrova, S. A. 1996, Pis'ma v AZh, 22, 445 [English translation: Astron. Lett., 22, 399]

Lyubarskii, Yu. E., \& Petrova, S. A. 1998, A\&A, 333, 181

Malofeev, V. M. 1999, Catalogue of Pulsar Radio Spectra (Pushchino: ACC FIAS)

Malofeev, V. M., \& Malov, I. F. 1980, AZh, 57, 90 [English translation: Sov. Astron. 24, 54]

Malofeev, V. M., Gil, J. A., Jessner, A., et al. 1994, A\&A, 285, 201

Malov, I. F. 1979, AZh, 56, 368

Malov, I. F., \& Malofeev, V. M. 1991, AZh, 68, 362

Manchester, R. N., \& Taylor, J. H. 1977, Pulsars (San-Francisco: Freeman)

McKinnon, M. 1997, ApJ, 475, 763

Petrova, S. A. 2000, A\&A, 360, 592

Petrova, S. A. 2001, A\&A, 378, 883

Petrova, S. A., \& Lyubarskii, Yu. E. 2000, A\&A, 355, 1168

Rankin, J. M. 1983, ApJ, 274, 359

Sieber, W. 1997, A\&A, 321, 519

Seiradakis, J. H., Gil, J. A., Graham, D. A., et al. 1995, A\&AS, 111,205

von Hoensbroech, A. G. 1999, Ph.D. Thesis, Max-PlankInstitut for Radio Astronomy, Bonn

Wielebinski, R., Jessner, A., Kramer, M., et al. 1993, A\&A, 272, L13

Xilouris, K. M., Kramer, M., Jessner, A., et al. 1996, A\&A, 309,481 